\title{
A Rare Case of Lance Adams Syndrome Post PEA Cardiac Arrest
}

\author{
Natasha Amjed, L Curtis Cary* \\ University of Tennessee College of Medicine Chattanooga \\ *Corresponding author: L Curtis Cary, University of Tennessee College of Medicine Chattanooga
}

\section{Introduction}

There is an increasing number of patients who have survived after suffering cardiac arrest. Some surviving patients experience prolonged neurological complications due to hypoxic brain injury, of which can result in acute post-hypoxic myoclonus and very rarely, Lance Adams Syndrome (LAS)5. LAS is clinically distinct from acute post-hypoxic myoclonus, which is much more common post-cardiac arrest. Patients who exhibit LAS typically have good outcomes. We present a patient post-cardiac who developed a rare case LAS who initially improved with treatment of levetracitram.

\section{Case Report}

A 72 year-old Caucasian male with a past medical history of hepatitis C, coronary artery disease status post percutaneous coronary intervention, poly-substance use, anxiety, hyperlipidemia, and depression was admitted to our emergency department after a witnessed cardiac arrest in the field. The patient was driving with a friend and reportedly pulled over after feeling unwell with subsequent cardiac arrest. He underwent 25 minutes of CPR before return of spontaneous circulation (ROSC) was achieved. While in the field, he was emergently intubated, received two amps of epinephrine, and one amp of bicarbonate. His initial rhythm was noted as pulseless electrical activity (PEA). An ECG was obtained and did not demonstrate ischemic changes. A subsequent bedside echocardiogram revealed decreased left ventricular ejection fraction. The Cardiac Arrest Hospital Prognosis (CAHP) score was 168 , indicating an $80 \%$ mortality rate with likely no meaningful neurological recovery. Aside from a gag reflex, the patient had no response to neurological stimuli. He underwent further evaluation and treatment per our institution's post-ROSC protocol work-up including a CT brain showing no acute intracranial abnormalities, targeted temperature management (TTM) for 24 hours, and administration of a heparin drip with plans for possible coronary angiography if subsequent neurological improvement was seen. He was hemodynamically stable while on mechanical ventilation, labs were within normal limits, and rapid SARS-CoV-2 testing was negative. Subsequent to TTM the patient's neurological status improved. The etiology of his cardiac arrest remained unclear.

By hospital day 10, the patient was more alert and was able to follow intermittent commands. He also developed persistent myoclonic jerking without correlating electroencephalogram (EEG) changes. Multiple EEGs were conducted throughout his hospitalization. His initial EEG was consistent with an abnormal coma EEG due to the presence of continuous generalized periodic discharges with associated time synched myoclonic jerks as well as polymorphic delta waveforms and diffuse suppressions. His most recent study was an abnormal awake and drowsy EEG which demonstrated continuous left upper limb myoclonic jerks without definitive epileptiform and mixed fast theta frequencies. MRI brain without contrast was also performed and revealed no acute findings with only mild chronic microangiopathic changes seen. By hospital day 11 , his neurological exam modestly improved as he was able to follow some commands and nod appropriately to questions. He continued to have upper and lower bilateral extremity myoclonus He received a trial of clonazepam without significant improvement of his myoclonus. The patient's family elected to proceed with trial of extubation which the patient passed with only a need for continued supplemental oxygen. His myoclonus worsened with external stimuli and interaction but improved with rest and sleep. His condition was thought to be consistent with an improving hypoxic ischemic encephalopathy and Lance Adams Syndrome. Given that clonazepam did not lead to meaningful improvement in his myoclonus, a trial of levetracitram $1000 \mathrm{mg}$ IV was administered leading to marked improvement in neurological status the following day. On hospital day 14 the patient's respiratory status improved and he no longer required supplemental oxygen. He followed commands appropriately and was able to respond verbally to some questions. He was maintained on levetracitram $500 \mathrm{mg}$ twice daily. Unfortunately, on hospital day 16, the patient's went into hypoxic respiratory failure. His family opted to move forward with comfort care at this juncture and the patient passed. 


\section{Discussion}

Lance Adams Syndrome occurs within days to weeks subsequent to cardiac arrest and resuscitation. It is known to persistent after neurological recovery post-cardiac arrest. The condition is exceedingly rare with just under 200 cases reported in the literature to our knowledge [1-3]. It is further categorized as a chronic post-hypoxic myoclonus, whereas acute post-hypoxic myoclonus, also termed myoclonic status epilepticus, usually occurs within 12 hours, and is much more common. LAS was first discovered in the 1960's by Lance and Adams. They had found that several patients developed myoclonic jerks after anoxic events. The myoclonic jerking persisted even after neurological improvement and gain of consciousness. Interestingly, the jerking episodes were worsened with external stimuli and improved with rest, as seen with our patient [3]. Clinical presentation of this disease can be associated with cerebellar ataxia, postural imbalance and intellectual deficits. Myoclonic jerks tend to be aggravated by external stimuli, voluntary movements, and sound. They disappear during sleep or periods when there is minimal to no external stimuli [2]. The pathophysiology of LAS comprises a loss of serotonin and GABA5. In one retrospective study conducted in California, LAS was characterized as myoclonus with reactive, epileptiform EEG, preserved evoked potentials and brainstem reflexes treated with anti-epileptics1. However, myoclonus associated with LAS has been reported to not have consistent correlation with EEG findings. Brain PET scan has been used to assist with diagnosis. According to one study, some patients with LAS had increased glucose metabolism in the pontine tegmentum, ventrolateral thalamus, and mesencephalon3. CT and MRI brain imaging are not typically indicated to aid in diagnosis.

It is important to distinguish between myoclonus status epilepticus and LAS. The most critical difference between the two is the presence of coma. Presence of coma is required as part of the diagnosis in myoclonus status epilepticus (MSE), while patients with LAS are alert and conscious. The time course for MSE is acute and generally lasts $12-24$ hours. LAS is more chronic and can last weeks to months. Myoclonus in MSE is generalized and multifocal, while LAS is intentional myoclonus. Lastly, MSE usually involves ischemic brain injury with neuronal necrosis. LAS results from hypoxic brain injury without irreversible infarction [4]. Patients have action myoclonus, particularly in the limbs. Patients who have cardiac arrest due to respiratory etiology tend to have better outcomes. Based on the literature, LAS tends to be associated with respiratory causes [4]. In regards to treatment, there are no current established guidelines for LAS. As such, most patients are treated empirically with clonazepam and levetiracetam, among other medications. One case report found that clonazepam was more effective in reducing myoclonus than was levetiracetam [5]. Another study suggested that response to clonazepam may be less than optimal on occasion. One case found that baclofen significantly improved myoclonus and may be indicated as an alternative therapy [6]. Our case report highlights the importance of early recognition of the very rare disorder of Lance Adams Syndrome. Additionally, the use of levetiracetam was initially noted to have marked improvement in myoclonic jerking in our patient. LAS typically has good outcomes, and our case further highlights a rare disease process with an unlikely poor outcome.

\section{Reference}

1. Aicua Rapun I, Novy J, Solari D, Oddo M, Rossetti AO (2017) Early LanceAdams syndrome after cardiac arrest: Prevalence, time to return to awareness, and outcome in a large cohort. Resuscitation 115: 169-172.

2. Ilik F, Kemal Ilik M, Cöven I (2014) Levatiracetam for the management of Lance-Adams syndrome. Iran J Child Neurol 8(2): 57-59.

3. Lee HL, Lee JK (2011) Lance-adams syndrome. Ann Rehabil Med 35(6): 939-943.

4. Malhotra S, Mohinder K (2012) Lance-Adams syndrome: Difficulties surrounding diagnosis, prognostication, and treatment after cardiac arrest. Anesth Essays Res 6(2): 218-222.

5. Shin JH, Park JM, Kim AR (2012) Lance-adams syndrome. Ann Rehabil Med 36(4): 561-564.

6. Wicklein EM, Schwendemann G (1993) Use of clonazepam and valproate in patients with Lance Adams syndrome. J R Soc Med 86(10): 618.
This work is licensed under Creative Commons Attribution 4.0 License

To Submit Your Article Click Here:

Submit Article
DOI: $10.32474 /$ RRHOAJ.2021.06.000238



